war demgegenüber die Frage nach Geschlechterrollen im $\mathrm{Fa}$ milienrecht. Hier nahmen wir das reformierte Unterhaltsrecht unter die Lupe. Dabei ergaben sich vor allem die Frage nach der Reproduktion der binären Geschlechterordnung durch Recht einerseits und die Problematik einer zwar formal, nicht aber tatsächlich egalitären Rollenverteilung andererseits. Außerdem befassten wir uns mit der Frage, inwieweit Heteronormativität Ausdruck in unserer Rechtssprache findet. Nur vordergründig geschlechtsneutrale Konzepte beäugten wir kritisch und fragten daher: Wie stellt sich die Sozialvertragstheorie aus feministischer Sicht dar? Außerdem: Gibt es eine feministische Demokratietheorie? Und, hochaktuell: feministische Kapitalismuskritik!

Die Diskussionen zeigten uns, wie sehr die Lektüre der im weitesten Sinne feministischen Hintergrundtexte unseren Blick auf klassisch-juristische Texte wie Gerichtsurteile oder Zeitschriftenartikel veränderte. Ehemals als selbstverständlich hingenommene Vorverständnisse gerieten ins Zwielicht, was allgemein eine deutlich kritischere Haltung gegenüber dem „Selbstverständlichen“ nach sich zog. So hinterfragten wir einerseits vermeintlich (geschlechts-)neutrale theoretischer Kategorien wie auch dem Recht zugrundegelegte (antizipierte) Lebenswelten. Andererseits sind es freilich die rechtlichen Kategorien selbst, die ein bestimmtes Verständnis von Geschlechtlichkeit und/ oder Sexualität festschreiben (Personenstand, Ehebegriff) und damit anderen möglichen Identitäten die (rechtliche) Anerkennung versagen. Recht reproduziert auf diese Weise Heteronormativität unter dem Deckmantel einer angeblich vorgefundenen sozialen Wirklichkeit, auf die es zu reagieren und die es zu regulieren habe. Ergebnis unserer Lektüre war, dass Recht selbst als dieser sozialen Wirklichkeit zugehörig und dementsprechend als konstruiert wie auch veränderbar begriffen werden sollte.

Eine wiederkehrende und zentrale Frage war, wie wirklichkeitsfremd eine derartige feministische Kritik ist, gemessen am herrschenden juristischen Diskurs. Diese stellte sich vor allem auch deshalb, weil wir neben den wenigen einschlägigen juristischen Autorinnen und Autoren mehrheitlich auf Beiträge aus den Geistes- und Sozialwissenschaften zurückgreifen mussten, um Antworten auf die aufgeworfenen Fragen zu erhalten. Ist die Idee gesellschaftlicher Konstruktion von Geschlechterbildern und -rollen zu weit abseits vom Mainstream als dass sie in den juristischen Diskurs Eingang finden könnte? In gewisser Weise lieferte der Vergleich mit einem ähnlichen Lesekreis in Cambridge (UK) eine Antwort. Nicht nur nahmen dort zahlreiche Männer an den Treffen teil, auch wurden die feministischen Texte, etwa in Kursen zu Human Rights, ganz selbstverständlich in das allgemeine Curriculum eingebunden; mithin waren alle Studierenden mit ein wenig feministischer Literatur konfrontiert - nach unserer Kenntnis haben alle überlebt ...

\title{
Das Mentoring-Programm JurMeP der Universität Potsdam
}

\section{Jutta Wickenhäuser}

Wissenschaftliche Hilfskraft Mentoring-Progamm für Juristinnen, Universität Potsdam

\section{Gleichstellung als Staatsziel}

„Der Staat fördert die tatsächliche Durchsetzung der Gleichberechtigung von Frauen und Männern und wirkt auf die Beseitigung bestehender Nachteile hin." Seit 1994 ist die juristische Gleichbehandlung in Art. 3 Abs. 2 Satz 2 GG als Staatsziel verankert, was jedoch nicht automatisch zu einer faktischen Gleichstellung zwischen Frauen und Männern führt, wie sich im Studium und später im Beruf immer wieder zeigt. Frauen werden schlechter bezahlt - sie verdienen durchschnittlich 23 Prozent weniger als Männer in vergleichbaren Positionen - haben schlechtere Aufstiegschancen und häufig Schwierigkeiten, Beruf und Familie zu verbinden. Um mit diesen Erkenntnissen und Schwierigkeiten nicht erst nach dem Studium konfrontiert zu werden, wurde 2008 an der Potsdamer Juristischen Fakultät das „Mentoring-Programm für Jurastudentinnen der Universität Potsdam “ durch die damaligen Gleichstellungsbeauftragten Judith Schmidt und Birgit Tropp- mann ins Leben gerufen. Begeistert vom Frauenförderprogramm „Justitia“ der Albert-Ludwigs-Universität Freiburg und dank wertvoller Ideen und Anregungen seitens Justitia konnte das Programm zum Sommersemester 2008 starten.

\section{JurMeP}

Seit 2010 hat das Programm einen neuen, prägnanten Namen: „JurMeP - Juristinnen Mentoring Potsdam“. Das Programm hat aber nach wie vor die gleichen Inhalte und Ziele wie seit seiner Gründung: JurMeP gibt Studentinnen die Möglichkeit, sich schon während ihres Studiums der oben dargelegten Problematik bewusst zu werden und Strategien für ihre berufliche und private Zukunft zu entwickeln.

Der Aufbau des Mentoring-Konzepts basiert auf drei Säulen: der Individualbetreuung, den Gruppenangeboten sowie der sog. Netzwerkpflege, also dem Austausch untereinander. Bei der die Individualbetreuung erhält die Jurastudentin (Mentee) eine Juristin als Mentorin zugeordnet, mit der sie sich regelmäßig treffen und an deren Vorbild sie im Rahmen der langfristigen Betreuung lernen kann. Die Mentorin steht der Mentee mit beruflichem und persönlichem Rat zur Seite 
und unterstützt sie beim Treffen bewusster Entscheidungen. Die Gruppenangebote sollen den Mentees u. a. das Spektrum möglicher juristischer Berufe näher bringen. So bietet JurMeP z.B. jedes Semester ein sog. Berufsbildervortrag mit dem Titel „Frauen im Recht“ an der Fakultät an. Zu den Themen „Juristinnen im Völkerrecht", „Juristinnen in der Wirtschaft", „Juristinnen im Strafecht“ sowie zuletzt „Juristinnen im Medienrecht" konnten die teilnehmenden Studentinnen in der Vergangenheit bereits viele hochkarätige Juristinnen und deren Vita kennenlernen. Auch „soft-skills“ der Mentees sollen im Rahmen dieser Säule gestärkt werden - hierzu wurden in der Vergangenheit zahlreiche Rhetorik- und Bewerbungstrainings angeboten. Einen praktischen Einblick in das Berufsleben von Juristinnen bekommen die Mentees wiederum durch Kanzleibesichtigungen, den Besuch von Ministerien und anderer Orte, an denen Juristinnen arbeiten.

Schließlich spielt bei JurMeP auch der Netzwerkgedanke eine große Rolle. Die Mentees sollen Netzwerke als Chance begreifen. Deshalb werden im Rahmen des Potsdamer Mentoring-Programms für Jurastudentinnen regelmäßig Stammtische für Mentees und Mentorinnen angeboten, bei denen sich alle Teilnehmerinnen kennen lernen und austauschen können. Dem gleichen Zweck dienen die zum Ende jedes Semesters organisierten gemeinsamen Unternehmungen, beispielsweise ein gemeinsames Grillfest oder die Besichtigung einer kulturellen Einrichtung.

\section{Teilnahme an JurMeP}

Als Mentee teilnehmen kann man als Jurastudentin der Universität Potsdam ab dem 2. Semester (Sommersemester). Voraussetzung ist die Teilnahme an einem Wochenendworkshop zum gegenseitigen Kennenlernen und zur Einführung der neuen Teilnehmerinnen in das Programm. Die Teilnahme der
Mentees an diesem Einführungsworkshop erleichtert auch die darauffolgende Zuordnung von Mentorinnen zu den einzelnen Mentees (das sog. „matching“).

Mentorin kann jede Juristin ab dem 1. Staatsexamen werden und wir freuen uns über jede Bewerbung. Auf ihre Aufgabe bereiten wir die Mentorinnen im Rahmen einer zweistündigen Schulung vor.

\section{Unterstützer von JurMeP}

Besondere Unterstützung erfährt das Mentoring-Programm seitens der Fakultät durch Frau Prof. Dr. Assmann, die sich insbesondere für die Vortragsreihe „Frauen im Recht“ einsetzt, sowie dem Verein der Freunde und Förderer der Juristischen Fakultät der Universität Potsdam. Auch hat sich in der Vergangenheit eine fruchtbare Zusammenarbeit mit dem Deutschen Juristinnenbund und der Vorsitzenden des Landesverbandes Brandenburg, Ramona Pisal, etabliert. Im Rahmen der Kooperation von JurMeP und djb konnten bereits ein gemeinsamer Berufsbildervortrag sowie ein Bewerbungstraining für Mentorinnen durchgeführt werden.

\section{Fazit}

Das noch junge Programm hat sich an der Juristischen Fakultät fest etabliert und betreut derzeit ca. 40 Mentee-MentorinTandems, Tendenz steigend. Die Teilnahme an JurMeP ist eine Chance, sich frühzeitig und strategisch auf die nach wie vor zu konstatierenden Schwierigkeiten von Juristinnen auf dem Arbeitsmarkt vorzubereiten.

Das Organisationsteam um Winnie Locke, Judith Schmidt, Birgit Troppmann und Gundula Ziegenhagen sowie die Mitarbeiterinnen Laura Philipp und Jutta Wickenhäuser heißen jede Teilnehmerin willkommen, die diese Chance ergreifen möchte.

\section{Wer nicht wagt, der nicht gewinnt - Referendariat in Spanien}

\section{Katharina Miller, LL.M. \\ Rechtsanwältin/Abogada inscrita, Madrid}

Frei nach dem Motto „Desde Madrid al cielo”, was übersetzt werden kann mit „Nach Madrid ist nur der Himmel schöner”, habe ich mich gleich zu Beginn meines Referendariats in Saarbrücken dazu entschlossen, mich während der Referendarszeit in Madrid sowohl der Juristerei als auch der spanischen Sprache zu widmen. Denn nach einem Auslandsjahr in Winchester (England), einem LL.M. in der französischen Sprache sowie einem daran anschließenden Praktikum am EuGH in Luxemburg wollte ich nun auch noch die spanische Sprache erlernen.

Mein Ausbildungsrichter am EuGH kannte von seiner Zeit in Madrid die Anwaltskanzlei Dr. Frühbeck Abogados, S.L.P., weshalb meine an diese Kanzlei gerichtete Bewerbung für die nach $\mathbb{} 24$ der saarländischen JAG geregelte Rechtsanwaltsstation II nahe lag. Sowohl die Kanzlei als auch ich gingen davon aus, dass meine Spanischkenntnisse in der Zwischenzeit nachgeholt werden konnten, da ich theoretisch noch 1 1/2 Jahre Zeit zum Erlernen der Sprache hatte. Für die anschließende Wahlstation bekam ich bei der Gesellschaft für technische Zusammenarbeit (gtz) in Lima eine Zusage. Dort hätte ich in einem Reformprojekt des peruanischen Justizwesens mitarbeiten können. Doch der deutsche Konjunktiv ist hier sehr aussagekräftig, denn am Ende kam alles anders.

So landete ich am Flughafen Madrid-Barajas ohne Spanischkenntnisse. Zu schnell war die Referendarszeit vorübergegangen, zumal ich ein Semester an der Verwaltungshoch- 